\title{
Analgesic effects of bilateral superficial cervical plexus block in Thyroid Operation
}

Mónica Nunes Ferreira ${ }^{1}$, Joana Sousa Correia¹, Ana Afonso', Germano Cardoso'

${ }^{1}$ Department of Anaesthesiology and Intensive Care, Instituto Português de Oncologia, Porto, Portugal

Euroanaesthesia

\section{INTRODUCTION}

Bilateral superficial cervical plexus block (BSCPB) is a simple non-invasive technique that can be used as analgesic measure in the perioperative period of thyroidectomy $[1,2]$.

\section{PURPOSE}

The purpose of this study is to assess the analgesic effects of this technique during the intraoperative and postoperative periods of thyroid operations and to determine whether it reduces the adverse effects of general anesthesia.

\section{METHODS}

- A retrospective study was conducted between January-October 2016 in a singlecenter.

- 42 patients elective thyroidectomy

- 42 included in the study

- In all the included patients was performed: intravenous induction with fentanyl, propofol and rocuronio, intraoperative analgesia with fentanyl and paracetamol, and maintenance with desflurane.

- The following variables were analyzed:

- Amount of opioid consumed during surgery;

- Postoperative pain (the time to the first analgesics required and amount of opioid consumed in the recovery room)

- Recovery room stay

- Postoperative nausea and vomiting in the recovery room

15 BSCPB

[after the induction of general anaesthesia]

- $5 \mathrm{~mL}$ of $1 \%$ Ropivacaine per side

- We used SPSS (PSAW Statistics 18) for statistical analysis, non-parametric tests were performed, and a value of $p<0.05$ defined as a statistically significant result.

\section{RESULTS/DISCUSSION}

- There were no significant differences in patient characteristics

- It was found that the BSCPB group consumed fewer opioids during surgery $(p<0.001)$ (average consumption of fentanyl in milligrams (mg): BSCPB 0.11 vs SA 0.28) (Graphic 1).

- We observed a longer duration of analgesia in BSCPB group $(p=0.003)$ (average of time in minutes (min) BSCPB 38.3 vs SA 8.9) ) (Graphic 2) and a smaller amount of morphine consumed in recovery room ( $p=0.018$ ) (average consumption in mg: BSCPB 0.4 vs SA 2.1) (Graphic 3) .

- The recovery room stay was fewer in BSCPB group $(p=0.021$ ) (average of time in min: BSCPB 50.0 vs SA 58.9) (Graphic 4).

- No statistically significant differences in the remaining variables.

- It should be noted that there were no complications in both groups.

\section{CONCLUSION}

BSCPB reduces analgesic use in intra and postoperative periods of thyroid operations. It also significantly shortens the recovery room stay.
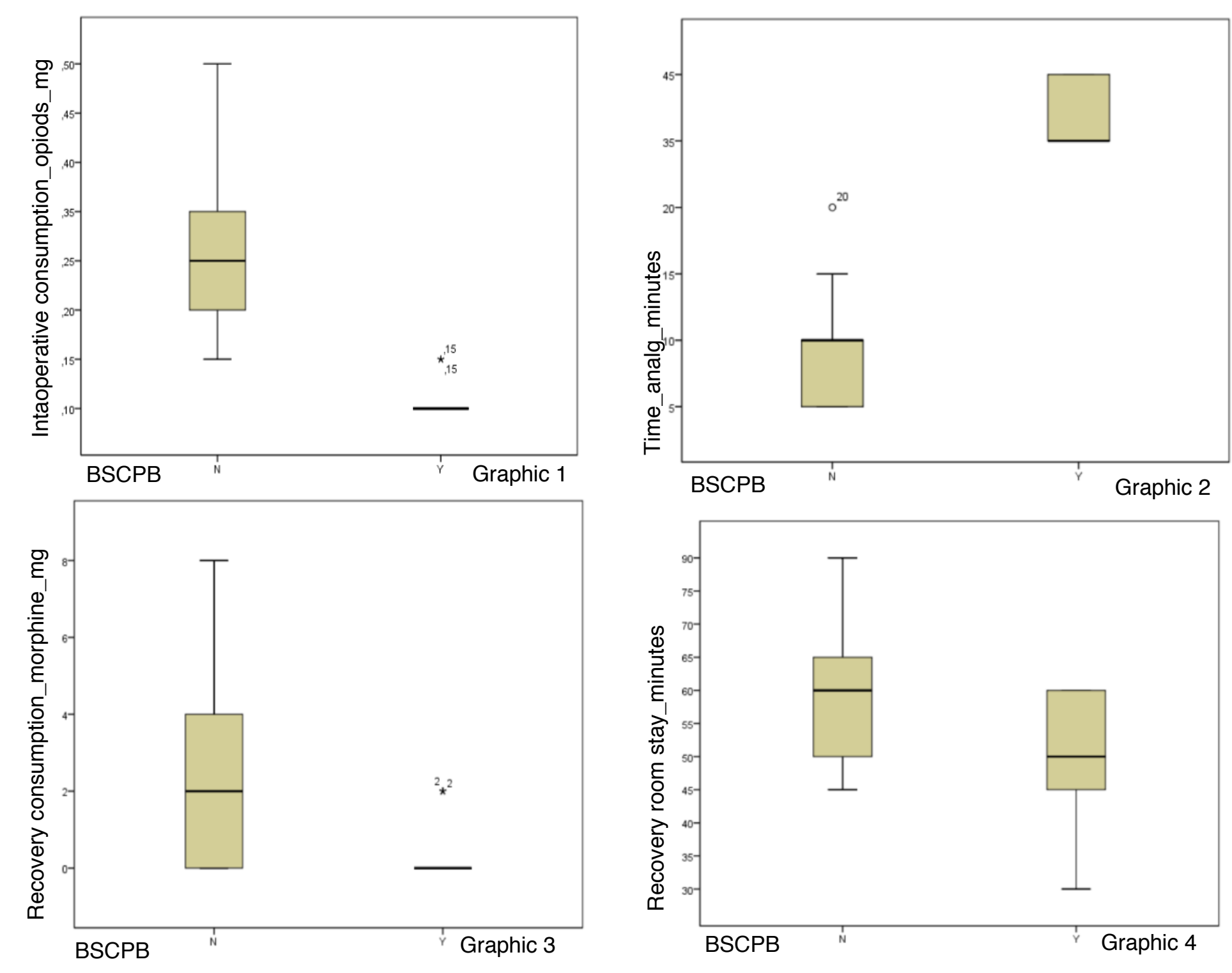

\section{REFERENCES}

1. Rev Chil Cir. 2014; 66: 531-535

2. World J Surg. 2010; 34: 2338-2343 